\title{
Achievable Rates of Cognitive Radio Networks Using Multi-Layer Coding with Limited CSI
}

\author{
Lokman Sboui, Student Member, IEEE, Zouheir Rezki, Senior Member, IEEE, and Mohamed-Slim \\ Alouini, Fellow, IEEE. \\ King Abdullah University of Science and Technology (KAUST), \\ Computer, Electrical and Mathematical Science and Engineering Division (CEMSE), \\ Thuwal 23955-6900, Saudi Arabia. \\ \{lokman.sboui, zouheir.rezki, slim.alouini\}@kaust.edu.sa
}

\begin{abstract}
In a Cognitive Radio (CR) framework, the channel state information (CSI) feedback to the secondary transmitter (SU_Tx) can be limited or unavailable. Thus, the statistical model is adopted in order to determine the system performance using the outage concept. In this paper, we adopt a new approach using multi-layer-coding (MLC) strategy, i.e., broadcast approach, to enhance spectrum sharing over fading channels. First, we consider a scenario where the secondary transmitter has no CSI of both the link between SU_Tx and the primary receiver (cross-link) and its own link. We show that using MLC improves the cognitive rate compared to the rate provided by a singlelayer-coding (SLC). In addition, we observe numerically that 2-Layer coding achieves most of the gain for Rayleigh fading. Second, we analyze a scenario where $\mathrm{SU}_{-} \mathrm{Tx}$ is provided by partial CSI about its link through quantized CSI. We compute its achievable rate adopting the MLC and highlight the improvement over SLC. Finally, we study the case in which the cross-link is perfect, i.e., a cooperative primary user setting, and compare the performance with the previous cases. We present asymptotic analysis at high power regime and show that the cooperation enhances considerably the cognitive rate at high values of the secondary power budget.
\end{abstract}

Index Terms-Cognitive radio, broadcast approach, achievable rate, quantized CSI, fading channels.

\section{INTRODUCTION}

The Cognitive radio (CR) is a promising concept that aims to overcome the spectrum scarcity issue [2] in order to cope with the evolution of wireless communications towards next generation, e.g. 5G [3]. This concept is based on including an unlicensed/secondary user (SU) that shares the spectrum of a licensed/primary user (PU) without affecting the primary communication. One of the $\mathrm{CR}$ strategies is the underlay CR [4], where the PU tolerates a simultaneous secondary communication subject to an interference threshold, the SU must adapt its power so that the caused interference is below this threshold. In this setting, the knowledge of the SU-PU channel state information (CSI) is crucial in order to protect the primary communication conveniently [5]. In the same time, the CSI of the secondary is also important to efficiently exploit spectrum sharing and to maximize the cognitive rate.

Part of this work has been accepted for presentation at the 2013 Global Communications Conference (Globecom'2013), Atlanta, GA, USA [1].

Copyright (C) 2015 IEEE. Personal use of this material is permitted. However, permission to use this material for any other purposes must be obtained from the IEEE by sending a request to pubs-permissions@ieee.org.
However, assuming perfect CSI is based on the fact that the coherence time of the fading channel spans many coherence blocks so that the channel can be estimated at the receiver side with minimal overhead used in the pilot [6]. If in addition, no delay constraint is imposed and communications happens over many fading channel coherence blocks, the maximum achievable rate that can be obtained is the ergodic capacity. In this case, it is well-known that the optimal coding strategy consists of using a fixed-power fixed-rate Gaussian codebook where each codeword is scaled by the total available power constraint. In the case where perfect CSI at the transmitter is available, the optimal coding strategy consists of using either a multiplexed multiple codebook scheme [7] or a simple constant-rate constant-power Gaussian codebook where the code symbols are scaled dynamically by the appropriate power allocation function before transmission [8]. However, when a delay constraint is to be respected, the notion of capacityversus-outage is more appropriate and without perfect CSI at the transmitter. The capacity in the Shannon sense is equal to zero since no matter how small the rate we are communicating at, there is no guarantee that this rate can be conveyed reliably to the receiver [9], [10]. For most cases, CSI at the transmitter gives an advantage only through power control. While this advantage is generally not significant under a short term power constraint, it is remarkably considerable under long term power constraint, see e.g., [11]-[15] and references therein. Therefore, we focus on communication scenarios where the transmitter is either only aware of the statistics of the fading channel or is provided with a quantized version of the actual channel gain through an error-free lowrate feedback link [16]. As the feedback link is of low rate, one can reasonably justify the error-free assumption of the feedback. The multi-layer coding (MLC) for a compound channel using the broadcast approach (BA) has been first introduced by Cover [17]. This approach facilitates to deliver information rates which depend on the actual realization of the channel and without the transmitter being aware of which state the channel is. The BA has been pursued in the case of flat-fading Gaussian channel with no fading dynamics, i.e., the quasi-static channel model, in [18]. The quantized CSI mechanism is described as follows: the continuous channel gain is subdivided into a finite number of channel regions and the feedback, [16], from the receiver indicates in which region the actual channel is, this concept helps in dealing with the 
channel as parallel broadcast channels [19], [20]. Adopting MLC in CR is expected to enhance the achievable rate when the CSI is either not available or limited through quantized knowledge. In fact, in [19], the authors have studied a point to point scheme and have shown that MLC enhancement of the rate becomes limited when the quantized CSI feedback rate is high. From another side, the cooperation of the PU was studied in [21]-[23], where the authors adopted a cooperative ZF beamforming in a multiuser multiple-input single-output (MISO) scenario and showed that the system performances depend on the CSI feedback.

In this paper, we study the achievable rate of a spectrum sharing setting using a broadcast approach. We examine the impact of various CSI assumptions at the secondary transmitter on the achievable rate. We argue that this scenario has not yet been given full consideration in existing state of the art. Our purpose is to maximize the cognitive achievable rate and understand how the MLC would perform in CR context when the CSI is not available or limited. This scenario corresponds to a more realistic situation where the secondary link can be estimated up to certain accuracy and where the primary user (PU) receiver tolerates some interference from the secondary user (SU) (most probably to get some rewards). We, then, present the interpretation of single-user MLC e.g., [24], [25] and we focus on the eventual improvement on the achievable rate gained compared to the throughput provided by single layer coding (SLC). In addition we study the secondary rate when quantized CSI feedback is provided and a BA is adopted. Our objective is to go further and analyze the MLC effect in a CR setting where an additional interference temperature constraint is considered to protect the PU. We believe that such study is relevant to CR especially when the interference constraint affects the rate at high power budget as shown in [26]. In addition, we evaluate the validity of MLC with cross link CSI availability (through primary cooperation) and assess whether MLC is beneficial or not to the SU [27]. The main contributions of this work consist of

- Computing the cognitive rate using 2-layer coding and MLC and highlighting its eventual improvement compared to outage capacity.

- Determining the effect of quantized CSI feedback and MLC coding on cognitive rate.

- Developing heuristic algorithms to solve non-convex optimization problems describing the cognitive rate with MLC and quantized CSI.

- Analyzing the impact of PU cooperation on the cognitive rate when MLC is adopted.

- Presenting an asymptotic analysis of the cognitive rate at high power regime.

The rest of the paper is organized as follows. In Section II, the system model is presented. In Section III, we compute the cognitive average throughput using MLC and assuming statistical CSI of both the secondary link and the cross link. Section IV highlights the effect of the cross link CSI on the secondary rate and presents results where only quantized cross link CSI is available at the secondary transmitter. Asymptotic analysis is presented in Section VI. Numerical results and their

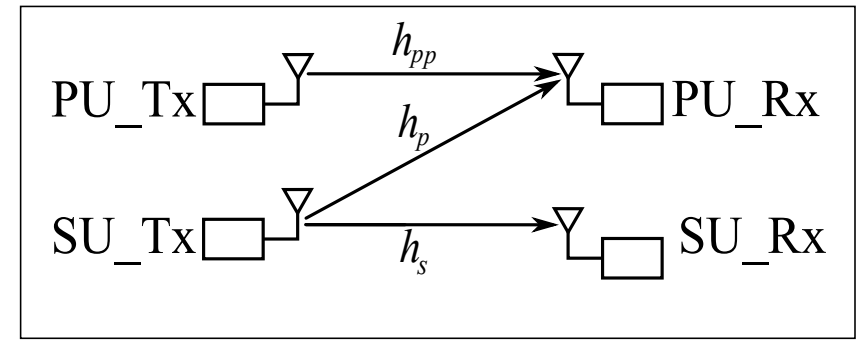

(a)

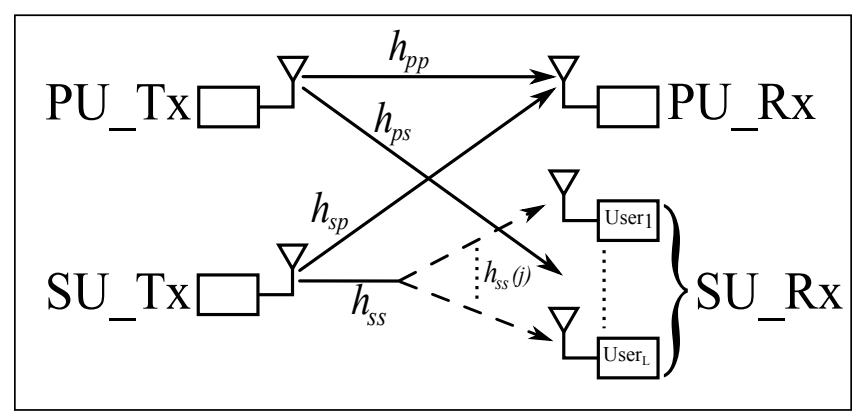

(b)

Figure 1: (a) A spectrum sharing channel model (b)

Equivalent model adopting the broadcast approach.

interpretations are presented in Section VII. Finally, the paper is concluded in Section VIII.

\section{System Model ANd Preliminaries}

\section{A. System Model}

We consider a spectrum sharing communication scenario as depicted in Fig.1(a), where a secondary user transmitter (SU_Tx) is communicating with a secondary user receiver (SU_Rx), under certain constraints that will be defined later, through a licensed bandwidth occupied by a primary user (PU). The signal received at the $\mathrm{SU}$ is given by:

$$
y_{s}(i)=h_{s} x(i)+n_{s}(i),
$$

where $i$ is the discrete-time index, $i=1, \ldots, T$, where $T$ is the coherence time assumed here equal to the symbol block length, for simplicity; the symbol $x(i)$ represents the channel input, $h_{s}$ is the complex channel gain, and $n_{s}(i)$ is a circularly symmetric complex Gaussian noise with zero mean and unit variance, i.e., $\mathcal{C N}(0,1)$ and is independent of $h_{s}$. The modulus squared channel gains $\left|h_{s}\right|^{2},\left|h_{p}\right|^{2}$ and $\left|h_{p p}\right|^{2}$ are assumed to have continuous probability distribution function (PDF)'s denoted $f_{\mathbf{h}_{s}}\left(h_{s}\right), f_{\mathbf{h}_{p}}\left(h_{p}\right)$ and $f_{\mathbf{h}_{p p}}\left(h_{p p}\right)$, respectively. We consider a short term power constraint, $P_{S T}$ (excluding power adaptation over different transmission blocks). The channel model (1) is also known as the quasi-static flat fading channel model suitable for delay-constrained communications. The SU_Tx is ignorant about the instantaneous CSI of the SU link (CSI-SL), $h_{s}$. However, it is provided its statistics through $f_{\mathbf{h}_{s}}\left(h_{s}\right)$. For the CSI of the cross link (CSI-CL), we analyze two scenarios: non-cooperative and cooperative. In non-cooperative scenario, the PU does not provide any feedback about the CSI-CL to 
the SU_Tx. Thus, the interference/cognitive constraint on the SU power is statistical. In the cooperative scenario, the PU is willing to share the CSI-CL with the SU_Tx and the cognitive constraint becomes instantaneous and the transmit power can be adapted with the instantaneous channel gain $h_{p}$. Without cognitive constraint, the classical coding strategy consists of a single-codebook, on-off power transmission scheme. This is known as the outage approach (OA) (also called outage capacity) or SLC [28]. Comes the cognitive constraint (either statistical or on the average), several authors have adopted such an approach with proper adaptation to CR settings, e.g., [10], [29]. To the best of our knowledge, performance of MLC in CR settings has not been evaluated previously which constitutes the main motivation of this paper.

\section{B. Preliminary}

In this preliminary, we briefly describe MLC in fading channels, more details can be found in [18]. An equivalent broadcast channel of the secondary link is illustrated in Fig.1(b). During each channel realization $h_{s}$, the transmitter sends infinite/finite number of layers of coded information. The receiver is regarded as continuum of ordered users, each decoding a code layer if the channel realization allows it. The ordering here is guaranteed by the nature of the Gaussian broadcast channel. Each of the users has to decode a fractional rate, $d R$, which is not equal for different users and rather depends on the user index. The first user (the weakest of all users or equivalently the one supporting the lowest data rate) decodes only its own $d R$, the second user, since supporting higher data rate, decodes first the information intended to the first user, cancels it out and then decodes its own $d R$. The third user begins by decoding data information designated to users 1 and 2 , and then decodes its own data rate $d R$, and so on so forth until user $j$. The broadcast strategy relies on this fact and assumes that there is an infinite/finite number of ordered receivers. In the model (1), each realization of $h_{s}$, is associated to an achievable rate and we are interested in the average achievable rate for various independent transmission blocks. Obviously, if perfect CSI is available at the transmitter, the expected rate over many transmission blocks and under a short term power constraint $\underset{x}{\mathbb{E}}\left[|x|^{2}\right] \leq P_{S T}$, is given by:

$$
C_{e r g}=\underset{\gamma_{s}}{\mathbb{E}}\left[\log \left(1+P_{S T} \gamma_{s}\right)\right],
$$

where $\gamma_{s}=\left|h_{s}\right|^{2}$. Since the actual channel realization is $h_{s}^{(j)}$, then any rate slightly higher than $\log \left(1+P_{S T} \gamma_{s}^{(j)}\right)$ cannot be decoded reliably and thus users $j+1, j+2, \ldots$ achieve nothing. Hence, the total achievable rate for channel realization $h^{(j)}$ is the integral of $d R$ over all users up to $j$. While the number of code layers depends on the cardinality of the fading power random variable $\gamma_{s}$, a practical finite number of code layers may be used to tradeoff performance versus encoding/decoding complexity.

\section{Non-CoOperative MLC WITH No CSI-SL}

This setting describes a spectrum sharing communication where, in the absence of a potential incentive and/or a cooperation protocol, the PU is not willing to feed back the channel gain $h_{p}$ to the SU_Tx. It is clear that in the absence of the cross link CSI, the SU_Tx is unable to protect fully the primary receiver (PU_Rx) against interference. Instead, the SU relies on the statistics of $h_{p}$ to establish a reliable communication without prejudicing the PU [30]. That is, the probability that the interference power at the PU be above a given positive threshold should be kept as small as possible, i.e.,

$$
\operatorname{Prob}\left\{P \gamma_{p} \geq Q_{\text {peak }}\right\} \leq \varepsilon,
$$

where $P$ is the transmit power at the SU_Tx, $\gamma_{p}=\left|h_{p}\right|^{2}$, $Q_{\text {peak }}$ is the interference level tolerated by the PU_Rx and $\varepsilon>0$ is a threshold that may be regarded as the percentage of time that the SU is allowed to exceed $Q_{\text {peak }}$. Constraint (3) aims at reducing the instantaneous interference power at the PU_Rx for a given secondary link channel realization $h_{p}$. We could have set (3) more reliably as a constraint on the Signal to Interference plus Noise Ratio $(S I N R)$ at the PU_Rx, however this requires the knowledge of both the power at the primary transmitter (PU_Tx) and the statistics of the primary link $h_{p p}$. Note that (3) is equivalent to:

$$
P \leq P_{\text {peak }}
$$

where $P_{\text {peak }}=\frac{Q_{\text {peak }}}{F_{\mathbf{h}_{p}}^{-1}(1-\varepsilon)}$ and $F_{\mathbf{h}_{p}}^{-1}(\cdot)$ is the inverse cumulative distribution function (CDF) of $\left|h_{p}\right|^{2}$. Constraint (4) can be interpreted as a peak transmit-power constraint dictated by the interference constraint of the PU_Rx. Meanwhile, the short term power constraint is written as

$$
P \leq P_{S T}
$$

Consequently, (4) and (5) can be written as

$$
P \leq \min \left(P_{S T}, P_{\text {peak }}\right) \triangleq \mathbb{P}_{0} .
$$

For the CSI-SL, we consider 2 cases; a case in which the SU is unable to estimate its channel and a case when it is provided a limited/quantized feedback from the SU_Rx. Note that in the regime where $P_{S T}>P_{\text {peak }}$, we have $P^{*}=P_{\text {peak }}$. Consequently, the SU has to use a power $P$ that does not exceed $P_{\text {peak }}$ to avoid power waste with no rate gain. When no CSI is available, the SU_Tx try to apply a MLC in order to increase its rate. We first present an extreme case of infinite layer coding. Then, we study a special finite layer coding with 2 layers.

\section{A. Infinite Layer Coding}

For a continuous fading power, the total achievable rate for a fading realization $\gamma_{s}=\left|h_{s}\right|^{2}$ is the integration over all the fractional achievable rates of all fictive users with successful layer decoding:

$$
R\left(\gamma_{s}\right)=\int_{0}^{\gamma_{s}} \frac{u \rho(u)}{1+u I(u)} d u
$$

where $u \rho(u) d u$ is the receive power of a layer parameterized by $u$ and intended to the user indexed by the channel gain power $u$; and $I(u)$ is the interference induced by information data intended to users indexed by channel gain powers $v>u$. 
The later data streams cannot be decoded reliably and thus constitute an additional interference noise

$$
I\left(\gamma_{s}\right)=\int_{\gamma_{s}}^{\infty} \rho(u) d u
$$

In particular, the total available power at the transmitter is given by:

$$
P_{S T}=\int_{0}^{\infty} \rho(u) d u
$$

Therefore, the maximum average rate achieved by this broadcast approach over many transmission blocks, $R_{b a}$, is given by: $R_{b a}=\int_{0}^{\infty} R(u) f_{\mathbf{h}_{s}}(u) d u$. Maximizing the average rate over all transmit power strategies is formulated as follows:

$$
R_{b a}^{\max }=\max _{I(u)} \int_{0}^{\infty} R(u) f_{\mathbf{h}_{s}}(u) d u,
$$

This optimization problem has been solved in [18], where it is found that the optimal $I(u)$ is given by:

$$
I(u)= \begin{cases}\frac{1-F_{\mathbf{h}_{s}}(u)-u f_{\mathbf{h}_{s}}(u)}{u^{2} f_{\mathbf{h}_{s}}(u)} & \text { if } u_{0} \leq u \leq u_{1} \\ 0 & \text { else, }\end{cases}
$$

where $F_{\mathbf{h}_{s}}(u)$ is CDF of the fading power; and where $u_{0}$ is determined by $I\left(u_{0}\right)=P$ and $u_{1}$ by $I\left(u_{1}\right)=0$. From $(11)$, the optimal transmit power distribution $\rho\left(\gamma_{s}\right)$ can be derived using (8).

Therefore, the achievable rate using the BA holds with $P_{S T}$ substituted by $\mathbb{P}_{0}$. Even in CR settings, the BA outperforms the standard OA in terms of average achievable rate. Recall that the OA consists of transmitting at a fixed rate and whenever the channel power is above a certain threshold, successful decoding is possible, otherwise, an outage is declared. Clearly, the OA's average rate corresponds to the optimization (10) over a restrained subspace of transmit power policies defined by: $\rho\left(\gamma_{s}\right)=P \delta\left(\gamma_{s}-\gamma_{t h}\right)$, where $\gamma_{t h}$ is a threshold defining the outage event and where $\delta(\cdot)$ is the Dirac delta function.

Note that for real systems infinite layer coding can be costly in terms of processing. Hence, we analyze, next, a simpler MLC with only 2 layers.

\section{B. 2-Layer Coding}

Although the BA is formally described with infinite codelayers for fading with continuous power gains, the performance of few code-layers (e.g 2 layers) is very close to the optimal performance [19], [31]. To this end, we study the case of 2-layer coding. The SU_Tx sends a superposition of two codewords defined by two rates; $R_{1}$ and $R_{2}$ corresponding to the channels $\gamma_{1}$ and $\gamma_{2}\left(\gamma_{1} \leq \gamma_{2}\right)$, respectively. In this design, $\gamma_{1}$ and $\gamma_{2}$ are the reconstruction channels above which the signal is no more decodable. Each rate is rewarded by the probability that the actual channel gain is higher than $\gamma_{i}$, $i=1,2$, and the total rate is, hence, written as

$$
\begin{aligned}
R_{s, L=2}=\left[1-F_{\mathbf{h}_{s}}\left(\gamma_{1}\right)\right] & \log \left(1+\frac{\gamma_{1} P_{1}}{1+\gamma_{1} P_{2}}\right)+ \\
& {\left[1-F_{\mathbf{h}_{s}}\left(\gamma_{2}\right)\right] \log \left(1+\gamma_{2} P_{2}\right) . }
\end{aligned}
$$

where $P_{1}$ and $P_{2}$ are the powers allocated to each layer. By adopting a short term power and statistical interference constraints, these powers are subject to the following inequalities

$$
\begin{gathered}
P_{1}+P_{2} \leq P_{S T}, \\
\operatorname{Prob}\left\{\left(P_{1}+P_{2}\right) \gamma_{p} \geq Q_{\text {peak }}\right\} \leq \varepsilon .
\end{gathered}
$$

Recall that (13) and (14) can be reduced, as in (6), to:

$$
P_{1}+P_{2} \leq \mathbb{P}_{0}
$$

In order to find the problem solution, i.e optimal gains and powers that maximizes the rate (12), we define the following optimization problem:

$$
\begin{gathered}
\max _{\gamma_{1}, \gamma_{2}, P_{1}, P_{2}} R_{s, L=2}, \\
\text { subject to } P_{1}+P_{2} \leq \mathbb{P}_{0} .
\end{gathered}
$$

The solution of this problem cannot be determined using direct analytical optimization methods due to the non-convexity of the objective function and the dependency between all the parameters. Therefore, we proceed by a heuristic iterative algorithm that exploits the parallel broadcast channels results in [20]. A first step is to initialize the parameters with given values. Then, we use the classical Lagrangian method to find the optimal reconstruction channels $\gamma_{1}$ and $\gamma_{2}$. Recall that the Lagrangian function of the optimization problem (16) is defined by

$$
\mathcal{L}=R_{s, L=2}-\lambda\left(P_{1}+P_{2}-\mathbb{P}_{0}\right) .
$$

For given $\gamma_{1}$ and $\gamma_{2}$, the above optimization problem is equivalent to assigning powers to users in a fading broadcast channel where the probability of success of each layer is the reward assigned to each user [32]. We compute the partial derivatives of $\mathcal{L}$ with respect to $\gamma_{1}$ and $\gamma_{2}$ and determine their roots, respectively. Their general expressions are given by:

$$
\begin{aligned}
\frac{\partial \mathcal{L}}{\partial \gamma_{1}}=\left[1-F_{\mathbf{h}_{s}}\left(\gamma_{1}\right)\right] & \frac{P_{1}}{\left(1+\gamma_{1} P_{2}\right)\left(1+\gamma_{1}\left(P_{1}+P_{2}\right) P_{2}\right)} \\
& -f_{\mathbf{h}_{s}}\left(\gamma_{1}\right) \log \left(1+\frac{\gamma_{1} P_{1}}{1+\gamma_{1} P_{2}}\right) \\
\frac{\partial \mathcal{L}}{\partial \gamma_{2}}=\left[1-F_{\mathbf{h}_{s}}\left(\gamma_{2}\right)\right] & \frac{P_{2}}{1+\gamma_{2} P_{1}} \\
& -f_{\mathbf{h}_{s}}\left(\gamma_{2}\right) \log \left(1+\gamma_{2} P_{2}\right)
\end{aligned}
$$

Note that depending on the fading distribution, $\frac{\partial \mathcal{L}}{\partial \gamma_{i}}$ is easily expressed and determining $\gamma_{1}$ and $\gamma_{2}$ is performed using a simple root-finding algorithm. The second step is to find the optimal power values $P_{1}$ and $P_{2}$ given the new obtained values of $\gamma_{1}$ and $\gamma_{2}$. In the literature, i.e., [19], [32], similar optimization problems have been studied where the optimal power is determined with a method based on the water-filling power allocation. In fact, this optimization problem can be seen as allocating the total power $\mathbb{P}_{0}$ for a two-user Gaussian broadcast channel. In order to determine the optimal power allocation, we first define the index function $J(x)$ as

$$
J(x)=\arg \max _{i} \frac{1-F_{\mathbf{h}_{s}}\left(\gamma_{i}\right)}{\frac{1}{\gamma_{i}}-x}-\lambda, \text { for } x \in\left[0, \mathbb{P}_{0}\right],
$$


where $\lambda$ is the Lagrangian multiplier associated to the constraint (17). note that, $\lambda$ is a constant $\forall i$ and is computed such as

$$
\lambda=\max _{i} \frac{1-F_{\mathbf{h}_{s}}\left(\gamma_{i}\right)}{\frac{1}{\gamma_{i}}-\mathbb{P}_{0}} .
$$

For a given user $i$, the length of the set of $x$ such that $J(x)=i$ represent the fraction of the total power $\mathbb{P}_{0}$ allocated to this user as shown in [32]. Consequently the optimal power for each user is given by

$$
P_{i}^{*}=\{x \operatorname{such} \text { as } J(x)=i\}, \text { for } i=1,2 .
$$

Once $P_{1}$ and $P_{2}$ are computed using (23) we fix these values and compute the values of $\gamma_{1}$ and $\gamma_{2}$, using (19) and (20), in a new iteration until we reach the convergence of the rate (see Algorithm 1). Where $\epsilon_{0}>0$ determines the stopping

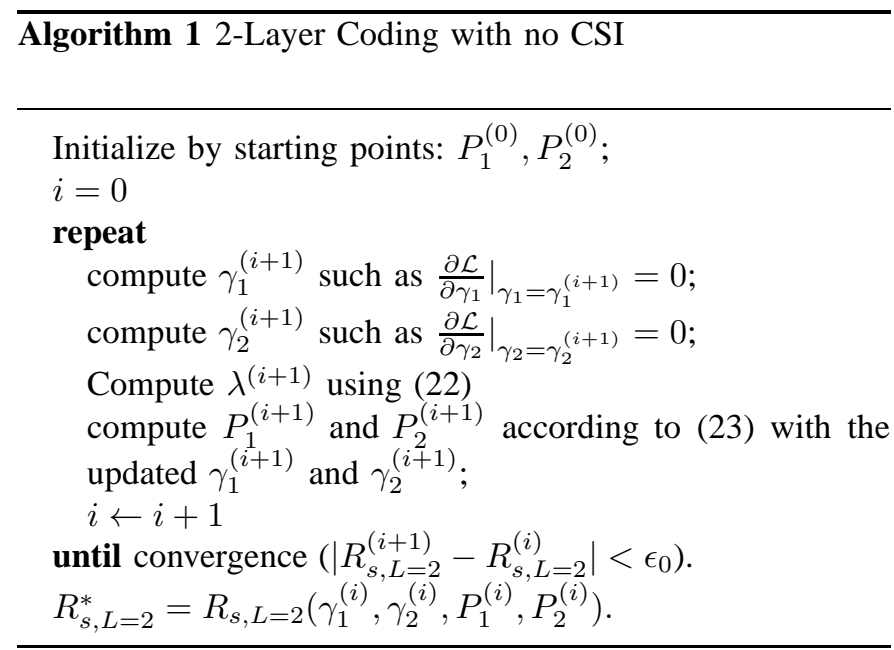

criteria of the algorithm. Note that the number of operations is linearly increasing with respect to the number of layers. Consequently, the complexity of our algorithms is also linear with the number of layers.

So far, we have assumed that the transmitter is not aware of the actual channel gain $h_{s}$. Clearly, this represents a worst case scenario that provides a lower bound on the average secondary throughput. However, in most wireless systems, there exists a feedback link from the receiver to the transmitter that may be used to provide a quantized version of the actual CSI to the transmitter [16]. Below, we study the impact of such a feedback on the performance of MLC in CR networks.

\section{Non-Cooperative MLC with Quantized CSI-SL}

The quantized feedback means that the SU_Tx receives, from the SU_Rx, the information that the actual channel realization is in a certain region among a set of $K$ predefined regions. This information requires a feedback link of rate $\left\lceil\log _{2}(K)\right\rceil$ bits per channel use. The relation between the number of feedback bits (NFB) and $K$ is $K=2^{N F B}$. Assume now that the channel gain set is subdivided into $\mathrm{K}$ regions $\left[\tau_{i-1}, \tau_{i}\right] i=1, \cdots, K$, where $\tau_{0}=0$ and $\tau_{K}=\infty$, for convenience. The fading channels with perfect CSI at the transmitter and at the receiver is generally well modeled by parallel Gaussian channels, where each of these channels represents a fading state [20]. With this approach, the degradedness of the additive white Gaussian noise (AWGN) broadcast channel is exploited in order to increase the expected cognitive rate. Although in our setting, we do not assume perfect CSI at the transmitter, SU_Tx looks at the reconstruction point $\gamma_{i}$ as being the actual channel gain in the region $i, i=1, \ldots, K$. Meanwhile, the SU_Rx performs a successive decoding scheme based on the rate of the actual channel gain. We first introduce the SLC with quantized CSI under a cognitive constraint as a benchmark. Then, we analyze the MLC with quantized CSI.

\section{A. Single-Layer Coding}

For a region of index $i, i=1, \ldots, K$, the SU_Tx uses the power $P$ to transmit the signal with a rate $R_{i}=\log \left(1+\gamma_{i} P\right)$, where $\gamma_{i}$ is the reconstruction point of region $i$. This strategy provides SU a reward equal to the probability of success, i.e., the probability that $\gamma_{i} \in\left[\tau_{i}, \tau_{i+1}\right]$. Consequently, by considering all the regions, the total expected rate of the SU, with quantized CSI, is determined by solving the following optimization problem

$$
\begin{aligned}
R_{s, K}= & \max _{\left\{P, \tau_{i}, \gamma_{i}\right\}} \sum_{i=1}^{K}\left[F_{\mathbf{h}_{s}}\left(\tau_{i+1}\right)-F_{\mathbf{h}_{s}}\left(\gamma_{i}\right)\right] \log \left(1+\gamma_{i} P\right) . \\
& \\
\text { s.t. } \quad & \tau_{i+1} \geq \gamma_{i} \geq \tau_{i}, \quad \text { for } i=1, \cdots, K . \\
& P \leq P_{S T} \\
& \operatorname{Prob}\left\{P \gamma_{p} \geq Q_{\text {peak }}\right\} \leq \varepsilon .
\end{aligned}
$$

To reduce the complexity of solving this problem, we determine the optimal values of some optimization variables. We first start by finding the optimal power $P^{*}$. From (26) and (27) we conclude that $P^{*}=\mathbb{P}_{0}$. Afterwards, the optimal $\tau_{i}$ 's that maximize $R_{s, K}$ are those which the probability of success is the highest, i.e., every $\tau_{i}$ should be the furthest from $\gamma_{i}$. Consequently, the optimal $\tau_{i}$ is the furthest from $\gamma_{i-1}$ as $F_{\mathbf{h}_{s}}(\cdot)$ is an increasing function. Hence, the optimal $\tau_{i}^{*}$ is such that

$$
\tau_{i}^{*}=\gamma_{i} \text { for } i=1, \cdots, K .
$$

Adopting (28) leads to a simpler problem with reduced number of variables. We, then, compute the Lagrangian of the problem as follows

$$
\mathcal{L}=R_{s, K}, \text { considering } P^{*}=\mathbb{P}_{0} \text { and } \tau_{i}^{*}=\gamma_{i},
$$

which gives the following optimality condition, for $i=1, \cdots, K$;

$$
\begin{aligned}
& \gamma_{i+1}^{*}= \\
& F_{\mathbf{h}_{s}}^{-1}\left(F_{\mathbf{h}_{s}}\left(\gamma_{i}^{*}\right)+f_{\mathbf{h}_{s}}\left(\gamma_{i}^{*}\right) \frac{1+\gamma_{i}^{*} \mathbb{P}_{0}}{\mathbb{P}_{0}} \log \left(\frac{1+\gamma_{i}^{*} \mathbb{P}_{0}}{1+\gamma_{i-1}^{*} \mathbb{P}_{0}}\right)\right)
\end{aligned}
$$

where $F_{\mathbf{h}_{s}}^{-1}(\cdot)$ is the inverse CDF of $\left|h_{s}\right|^{2}$. The condition in (30) are easy to solve since $\gamma_{i}$ can be expressed in function of $\gamma_{1}$, for $i=2, \cdots, K$. Finally, the rate $R_{s, K}$ is evaluated using the $\gamma_{i}^{*}$ 's (30). Note that in order to reach the perfect CSI rate, the number of quantized regions has to go to infinity for fading with unbounded support. 


\section{B. Multi-Layer Coding}

In this setting, in addition to the $K$ regions of channel gain, the SU_Tx transmits a superposition of $L$ codewords in each region. For a region of index $i, i=1, \cdots, K$, the rate of the codeword $j, j=1, \cdots, L$, corresponding to reconstruction channel gain $\gamma_{i j}$ is given by

$$
R_{i j}=\log \left(1+\frac{\gamma_{i j} P_{i j}}{1+\gamma_{i j} \sum_{k=j+1}^{L} P_{i k}}\right) .
$$

We assume that the set of $\gamma_{i j}$ 's is ordered such as $\gamma_{i 1} \leq \cdots \leq \gamma_{i L} \forall i=1, \cdots, K$. Recall that if the actual channel $\gamma_{s}$ is such that $\gamma_{s} \geq \gamma_{i j}$, then, the corresponding codeword is decoded successfully. Hence, the total secondary rate with MLC and quantized CSI is given by

$$
\begin{aligned}
R_{s, K, L}= & \max _{\left\{P_{i j}, \tau_{i}, \gamma_{i j}\right\}} \sum_{i=1}^{K} \sum_{j=1}^{L}\left[F_{\mathbf{h}_{s}}\left(\tau_{i+1}\right)-F_{\mathbf{h}_{s}}\left(\gamma_{i j}\right)\right] \times \\
& \log \left(1+\frac{\gamma_{i j} P_{i j}}{1+\gamma_{i j} \sum_{k=j+1}^{L} P_{i k}}\right), \\
\text { s.t. } \tau_{i+1} \geq \gamma_{i j}, \quad \gamma_{i 1} \geq \tau_{i}, \quad \gamma_{i(j+1)} \geq \gamma_{i j}, & \\
& \operatorname{Prob}\left\{\left(\sum_{j=1}^{L} P_{i j}\right) \gamma_{p} \geq Q_{\text {peak }}\right\} \leq \varepsilon, \\
& \sum_{j=1}^{L} P_{i j} \leq P_{S T}, \text { for } i=1, \cdots, K .
\end{aligned}
$$

Again, constraints (34) and (35) can be combined into a unique peak constraint as follows:

$$
\sum_{j=1}^{L} P_{i j} \leq \mathbb{P}_{0}=\min \left(P_{S T}, P_{\text {peak }}\right) .
$$

Note that, for the same reasons that have justified (28), the optimal set of $\tau_{i}^{*}$ can be set as

$$
\tau_{i}^{*}=\gamma_{i 1}, \quad \text { for } i=1, \cdots, K .
$$

Similarly to the single layer problem in Section IV-A a set of Karush Kuhn Tucker (KKT) optimality conditions are obtained after computing the Lagrangian. The optimality conditions are presented as follows; for $i=1, \cdots, K$

$$
\begin{aligned}
& \gamma_{(i+1) 1}^{*}=F_{\mathbf{h}_{s}}^{-1}\left(F_{\mathbf{h}_{s}}\left(\gamma_{i 1}^{*}\right)+f_{\mathbf{h}_{s}}\left(\gamma_{i 1}^{*}\right) \times\right. \\
& \left.\frac{\left(1+\gamma_{i 1}^{*} \mathbb{P}_{0}\right)\left(1+\gamma_{i 1}^{*}\left(\mathbb{P}_{0}-P_{i 1}\right)\right)}{P_{i 1}}\left(R_{i 1}-\sum_{j=1}^{L} R_{(i-1) j}\right)\right) .
\end{aligned}
$$

A similar problem was studied in [19] and the solution was computed using an iterative algorithm (See Algorithm 2). In Algorithm 2, we begin by choosing a set of $\gamma_{i 1}$ ' and power $P_{i j}$ 's as a starting point which gives $K$ MLC sub-problems. Then, for each region $i i=1, \cdots, K$ we compute the optimal set of $\gamma_{i j}(1<j \leq L)$ as in (30). The next step is to fix the $\gamma_{i j}$ 's and to compute the optimal $P_{i j}$ 's according to
(23). Finally, the optimality condition (38) is used to compute the optimal $\gamma_{i 1}$ 's when the $\gamma_{i j}$ and the $P_{i j}$ 's are fixed. Then we iterate until the maximum rate $R_{s, K, L}$ is obtained.

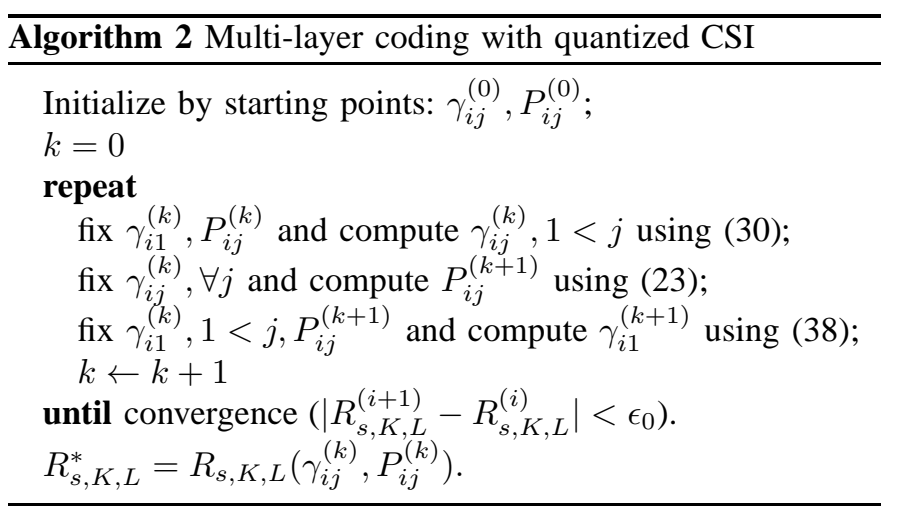

\section{Cooperative CR Broadcast Approach}

In cooperative $\mathrm{CR}$, the $\mathrm{PU}$ is willing to cooperate with the SU by providing the instantaneous CSI-CL [27]. The reason of cooperation is that the PU can increase the probability of transmission success by adopting the overlay CR strategy. In other words, the PU can cooperate with the SU and benefits from message relaying [33]. In our framework we focus on the cooperation from the SU perspective and we analyze how the BA scheme can improve the cognitive rate. In this scenario, the SU_Tx can fully protect the PU communication by limiting the instantaneous interference at the primary receiver. This interference constraint can be expressed as follows:

$$
P \gamma_{p} \leq Q_{\text {peak }} .
$$

We analyze the performance of this scheme similarly to the non-cooperative case. We investigate the use of MLC on the corresponding rate when the CSI-SL is either inexistent or limited.

\section{A. Cooperative MLC with No CSI-SL}

In this setting, the SU_Tx can obtain instantaneous CSI-CL form the PU_Rx, via a feedback link, but is not able to obtain CSI-SL due to the bad quality of its own channel, for instance.

1) Cooperative Infinite Layer Coding: When the SU adopts an infinite layer coding to enhance its rate in presence of the instantaneous CSI-CL, the corresponding power allocation is similar to the non-cooperative case since the interference constraint in (39) is a peak constraint and is similar to (4). In fact, we can express (39) as

$$
P \leq P_{\text {peak }}^{\prime}\left(\gamma_{p}\right),
$$

where $P_{\text {peak }}^{\prime}\left(\gamma_{p}\right)=\frac{Q_{p e a k}}{\gamma_{p}}$. Consequently, the resulting constraint of the power, considering both power and interference constraints, is

$$
P \leq \min \left(P_{S T}, P_{\text {peak }}^{\prime}\left(\gamma_{p}\right)\right) \triangleq \mathbb{P}_{1}
$$

Then, we compute the corresponding rate using $\mathbb{P}_{1}$ instead of $\mathbb{P}_{0}$ as in III-A for a given $\gamma_{p}$. The achievable rate is then obtained by averaging over all $\gamma_{p}$ realizations. 
2) Cooperative 2-Layer Coding: We study the 2-Layer Coding since it is simpler to implement than the infinite layer coding. The corresponding optimization problem is:

$$
\begin{aligned}
\max _{\gamma_{1}, \gamma_{2}, P_{1}, P_{2}} & R_{s, L=2}^{\text {Coop }}, \\
\text { s.t. } & P_{1}+P_{2} \leq P_{S T}, \\
& \left(P_{1}+P_{2}\right) \gamma_{p} \leq Q_{\text {peak }} .
\end{aligned}
$$

where $R_{s, L=2}^{C o o p}$ is given in (12). The constraints (43) and (44) can be written as a single constraint:

$$
P_{1}+P_{2} \leq \mathbb{P}_{1}
$$

Note that, this problem can be solved exactly as the problem in (16) by substituting $\mathbb{P}_{0}$ by $\mathbb{P}_{1}$ in Algorithm 1 for a given $\gamma_{p}$. The achievable rate is then obtained by averaging over many realizations of $\gamma_{p}$.

\section{B. Cooperative MLC with Quantized CSI-SL}

After studying the cooperative scenario when the CSI-SL is not available, we analyze the effect of quantized CSI-SL feedback under MLC when the PU is cooperative. We, first, start by the special case with single layer coding, SLC, then we generalize to MLC.

1) Cooperative Single-Layer Coding: The corresponding problem is given by:

$$
R_{s, K}^{C o o p}=\max _{\left\{P, \tau_{i}, \gamma_{i}\right\}} \sum_{i=1}^{K}\left[F_{\mathbf{h}_{s}}\left(\tau_{i+1}\right)-F_{\mathbf{h}_{s}}\left(\gamma_{i}\right)\right] \log \left(1+\gamma_{i} P\right) .
$$$$
\text { s.t. } \quad \tau_{i+1} \geq \gamma_{i} \geq \tau_{i} \quad \text { for } i=1, \cdots, K .
$$$$
P \leq P_{S T}
$$$$
P \gamma_{p} \leq Q_{\text {peak }} .
$$

The optimal reconstruction points are given similarly to the non-cooperative SLC problem and given by (28).

$$
\tau_{i}^{*}=\gamma_{i} \text { for } i=1, \cdots, K
$$

Then, the corresponding optimality conditions, for $i=1, \cdots, K$ is given by:

$$
\begin{aligned}
\gamma_{i+1}^{*}= & F_{\mathbf{h}_{s}}^{-1}\left(F_{\mathbf{h}_{s}}\left(\gamma_{i}^{*}\right)+\right. \\
& \left.f_{\mathbf{h}_{s}}\left(\gamma_{i}^{*}\right) \frac{1+\gamma_{i}^{*} \mathbb{P}_{1}}{\mathbb{P}_{1}} \log \left(\frac{1+\gamma_{i}^{*} \mathbb{P}_{1}}{1+\gamma_{i-1}^{*} \mathbb{P}_{1}}\right)\right)
\end{aligned}
$$

The rate $R_{s, K}^{c o o p}$ is optimally solved using the $\tau_{i}^{*}$ 's and the $\gamma_{i}^{*}$ 's form (50) and (51), respectively.

2) Cooperative Multi-Layer Coding: The cooperative MLC rate with quantized CSI given after solving the following optimization problem

$$
\begin{aligned}
R_{s, K, L}^{C o o p}= & \max _{\left\{\tau_{i}, \gamma_{i j}, P_{i j}\right\}} \sum_{i=1}^{K} \sum_{j=1}^{L}\left[F_{\mathbf{h}_{s}}\left(\tau_{i+1}\right)-F_{\mathbf{h}_{s}}\left(\gamma_{i j}\right)\right] \times \\
& \log \left(1+\frac{\gamma_{i j} P_{i j}}{1+\gamma_{i j} \sum_{k=j+1}^{L} P_{i k}}\right) \\
\text { s.t. } \quad & \tau_{i+1} \geq \gamma_{i j}, \quad \gamma_{i 1} \geq \tau_{i}, \quad \gamma_{i(j+1)} \geq \gamma_{i j} \\
& \gamma_{p} \sum_{j=1}^{L} P_{i j} \geq Q_{p e a k} \\
& \sum_{j=1}^{L} P_{i j} \leq P_{S T} \text { for } i=1, \cdots, K .
\end{aligned}
$$

The optimal region borders $\tau_{i}^{*}$ 's are again given as in (37) by

$$
\tau_{i}^{*}=\gamma_{i 1} \quad \text { for } i=1, \cdots, K .
$$

Then, after computing the Lagrangian, the optimality conditions, for $i=1, \cdots, K$, give

$F_{\mathbf{h}_{s}}\left(\gamma_{(i+1) 1}\right)=F_{\mathbf{h}_{s}}\left(\gamma_{i 1}\right)+$

$f_{\mathbf{h}_{s}}\left(\gamma_{i 1}\right) \frac{\left(1+\gamma_{i 1} \mathbb{P}_{1}\right)\left(1+\gamma_{i 1}\left(\mathbb{P}_{1}-P_{i 1}\right)\right)}{P_{i 1}}\left(R_{i 1}-\sum_{j=1}^{L} R_{(i-1) j}\right)$.

Finally, we adopt Algorithm 2 to compute the secondary achievable rate based on (51), (23) and (57) instead of (30), (23) and (38), respectively.

\section{Asymptotic AnALYsis}

We perform asymptotic analysis on the cognitive rate at high power regime to understand the best gain that could be achieved. The high power regime rate is defined as $\lim _{P_{S T} \rightarrow \infty} R_{s}$. Note that, when $P_{S T} \rightarrow \infty$, the power constraint becomes relaxed and does not affect the power allocation scheme. Consequently, the optimal power becomes only constrained by the interference constraint. Hence, our asymptotic study involves solving the previous problems excluding the power constraint.

\section{A. Non-Cooperative MLC}

In the non-cooperative MLC, when no power constraint is adopted, the optimal power is such that the interference constraint is fulfilled, i.e., $P^{*}=P_{\text {peak }}$ in the no CSI-SL case and $\sum_{j=1}^{L} P_{i j}^{*}=P_{\text {peak }}$ in the quantized CSI-SL case. That is, we let $\mathbb{P}_{0}=P_{\text {peak }}=\frac{Q_{\text {peak }}}{F_{\left|h_{p}\right|^{2}}^{-1}(1-\varepsilon)}$ and we use Algorithm 1 and Algorithm 2 to determine the asymptotic secondary rate.

\section{B. Cooperative $M L C$}

In cooperative scenario, as justified in the non-cooperative MLC we choose $\mathbb{P}_{1}=P_{\text {peak }}^{\prime}\left(\gamma_{p}\right)=\frac{Q_{\text {peak }}}{\gamma_{p}}$ and we compute the different asymptotes using the algorithms. Note that even if the asymptotes are determined by only removing the power constraint, its computation complexity is equivalent to solving the complete problem using the different algorithms once $Q_{\text {peak }}$ and $\epsilon$ are fixed. 


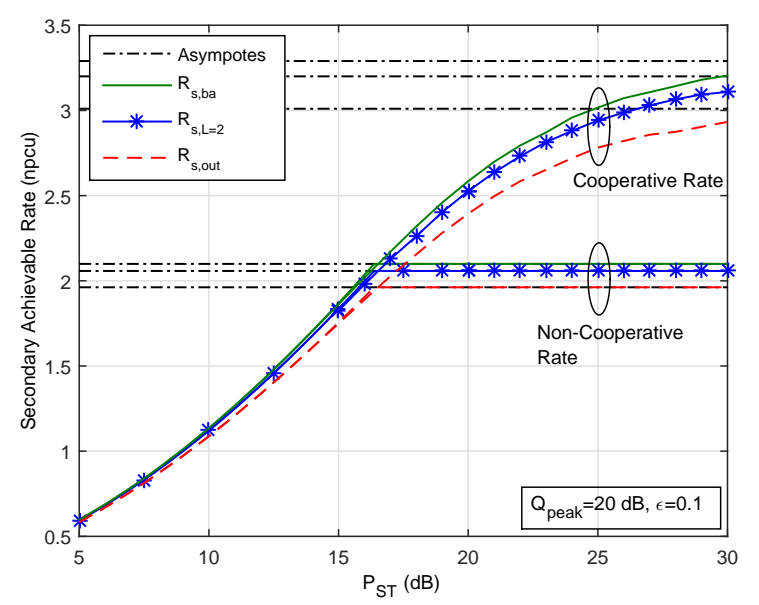

Figure 2: Secondary user average achievable rate using the broadcast approach, 2-layer coding and the standard outage approach.

\section{NUMERICAL RESULTS}

We apply the previous results to a normalized Rayleigh fading channel, i.e., $f_{\mathbf{h}_{s}}(x)=f_{\mathbf{h}_{p}}(x)=e^{-x}$ and $F_{\mathbf{h}_{s}}(x)=F_{\mathbf{h}_{p}}(x)=1-e^{-x}$.

The maximum average achievable secondary rate, using infinite layer coding with no cooperation, is given by [18]:

$R_{s, b a}^{n o c o o p}=R_{s, b a}^{\max }\left(\gamma_{0}\right)=2 E i\left(\gamma_{0}\right)-2 E i(1)-\left(e^{-\gamma_{0}}-e^{-1}\right)$,

where $\gamma_{0}=\frac{2}{1+\sqrt{1+4 \mathbb{P}_{0}}}$ and $\operatorname{Ei}(x)=\int_{x}^{\infty} \frac{e^{-t}}{t} \mathrm{dt}$ is the exponential integral function. In the case of cooperation, we have $R_{s, b a}^{c o o p}=R\left(\gamma_{1}\right)$ where $\gamma_{1}=\frac{2}{1+\sqrt{1+4 \mathbb{P}_{1}}}$. An evaluation of the secondary achievable rate using the $\mathrm{BA}, R_{s, b a}^{\max }$ given by (58) versus $P_{S T}$, is displayed in Fig. 2, for $Q_{\text {peak }}=20 \mathrm{~dB}$ and $\varepsilon=0.1$. Also we show, in Fig. 2, the average achievable rate using the standard OA, with $R_{s, \text { out }}^{\max }=e^{-\gamma_{t h}} \log \left(1+\gamma_{t h} \mathbb{P}_{0}\right)$, where $\gamma_{t h}$ is the outage threshold determined by solving the equation $\left(1+\gamma_{t h} P_{S T}\right) \log \left(1+\gamma_{t h} P_{S T}\right)=P_{S T}$.

From Fig. 2, we show that the BA outperforms the standard OA in terms of average achievable rate, especially at high SNR. This trends holds true for different values of $Q_{\text {peak }}$ and $\varepsilon$ with or without cooperation. Interestingly, even the less complex 2-layer coding $(\mathrm{L}=2)$ outperforms the classical $\mathrm{OA}$, as shown in Fig. 2. This expected result affirms that adopting the BA enhances the rate when no CSI is provided. In the cooperative setting, we show that the knowledge of the CSI-CL does not affect the secondary rate when $P_{S T}<P_{\text {peak }}=\frac{Q_{\text {peak }}}{F_{h_{p}}^{-1}(1-\varepsilon)}$. Hence, the instantaneous CSI-CL is not needed in this regime. However, if $P_{S T}>P_{\text {peak }}$, there is a remarkable gain in rate that can achieve $40 \%$ of the non-cooperation rate. Hence, cooperation is very rewarding in this case for the SU. In addition, in non-cooperative setting, there is no need that $P_{S T}$ exceeds $P_{\text {peak }}$ as discussed previously since there is no gain in the rate. However, in the cooperative setting, when $P_{S T}>P_{\text {peak }}$ the asymptotes are not reached even for $P_{S T}>30 \mathrm{~dB}$. We also show that when $P_{S T}$ increases, the gap between the rate in the broadcast and outage increases

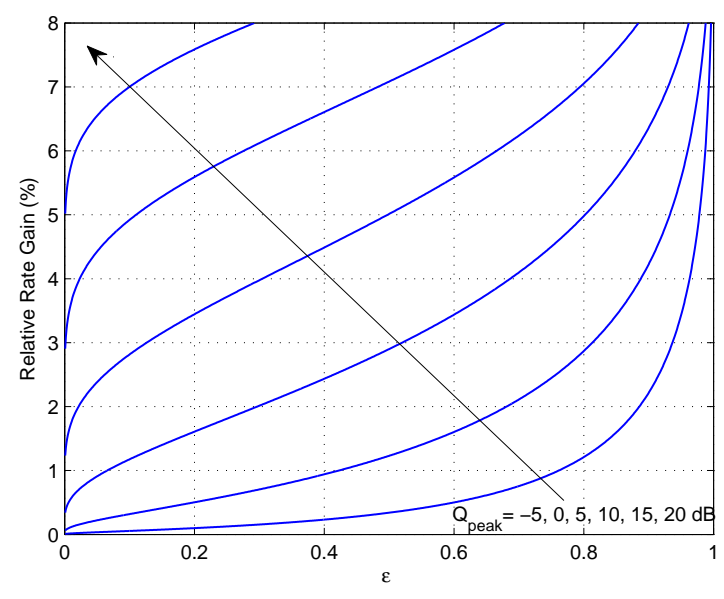

Figure 3: Relative rate gain percentage as function of $\varepsilon$ for different values of $Q_{\text {peak }}$ and $P_{S T}=20 \mathrm{~dB}$.

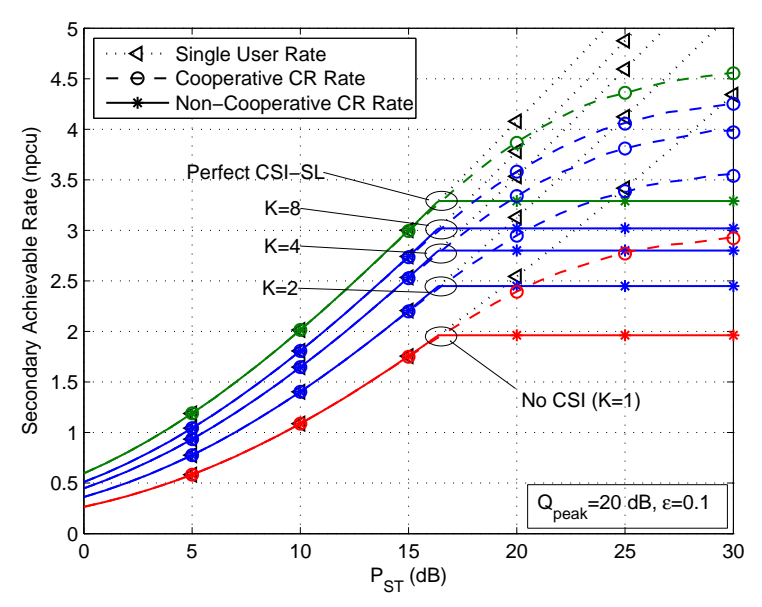

Figure 4: Achievable rate of the secondary user with single-layer coding and quantized CSI versus $P_{S T}$, for different number of feedback bits.

as well. The reason is that the effect of multi-layer coding is more clear at high SNR as it was shown in the numerical results (Fig. 2) of the reference [19].

Besides, in order to determine the effect of $Q_{\text {peak }}$ and $\varepsilon$ on the rate gain when adopting the $\mathrm{BA}$, we define a relative gain percentage as $\frac{R_{s, b a}-R_{s, \text { out }}}{R_{s, \text { out }}} \times 100$ presented in Fig.3 as a function of $\varepsilon$ for different values of $Q_{\text {peak }}$. We notice that for low values of $\varepsilon$ and $Q_{\text {peak }}$, the rate gain is increasing in a slow slope, whereas for high values of $\varepsilon$ and low values of $Q_{\text {peak }}$ the slope is quite sharp, the later observation also holds for low values of $\varepsilon$ and high values of $Q_{\text {peak }}$.

In Fig.4 the non-cooperative and cooperative achievable rates with SLC are presented as a function of $P_{S T}$, with $Q_{\text {peak }}=20 \mathrm{~dB}$ for different number of feedback bits: $N F B=0,1,2,3$ (i.e. $K=1,2,4,8$ ). We note that with quantized CSI feedback, the expected rate is enhanced but when the number of feedback bits increases, the rate gain 


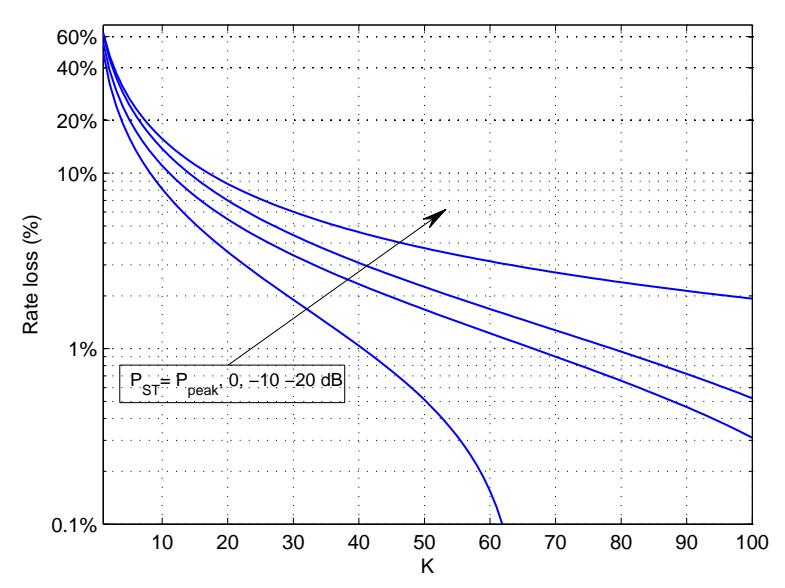

Figure 5: Secondary user rate loss percentage with quantized CSI versus the number of quantized region $K$.

becomes smaller as the perfect CSI rate (which is the upper bound) is becoming closer. In order to gain an insight about the convergence of the non-cooperative quantized CSI-SL rate, we define the percentage of rate loss, related to the perfect CSI-SL rate $R_{\text {perfectCSI }}$, as $R_{\text {Loss }}=\frac{R_{\text {perfectCSI}}-R_{s, b a}}{R_{\text {perfect } C S I}} \times 100$. This rate loss is presented as a function of the feedback bits number $\mathrm{K}$ in the OA context in Fig.5. We notice that achieving 90\% of the performance (which means a rate loss of 10\%) requires a relatively high number of feedback bits $N F B>3$ bits $(K>8)$. We, also, note that the value of the secondary power, $P_{S T}$, affects the convergence since for $P_{S T}=P_{\text {peak }}, 90 \%$ of the performance is achieved for $K=8$ i.e., $N F B=3$ bits whereas for $P_{S T}=-20 \mathrm{~dB}, K$ must be greater than 16 i.e., $N F B>4$ bits to achieve the target. The reason of fast convergence when $P_{S T}$ is high is that, at high SNR regime, the CSI knowledge is not needed to achieve the maximum rate as shown in [26], [34]. In Fig.6, the secondary rate as function of $P_{S T}$ with the quantized CSI and MLC is presented with and without cooperation in Fig.6(a) and Fig.6(b), respectively. We note that when K increases, the gain of MLC decreases. Hence, the availability of a quantized CSI at the transmitter undermines the performance gain of the MLC. As shown in Fig.6, there is no need for a MLC when K exceeds 4, since both $\mathrm{BA}$ and $\mathrm{OA}$ perform quasi similarly in this case.

\section{CONCLUSION}

In this paper, we have studied the achievable rate of the secondary link in a cognitive radio (CR) spectrum sharing scenario when a multi-layer coding (MLC) is adopted. First, with no primary user cooperation, i.e., the cross link channel is not provided, we use an infinite-layer coding, we have highlighted the corresponding improvement on the expected secondary rate when no CSI is available at the transmitter. We have, also, shown numerically that a 2-layer coding achieves most of this gain, for Rayleigh fading channels. Second, we have considered a non-cooperative situation where the secondary user transmitter is aware of a quantized version of its actual CSI, and evaluated the average throughput.

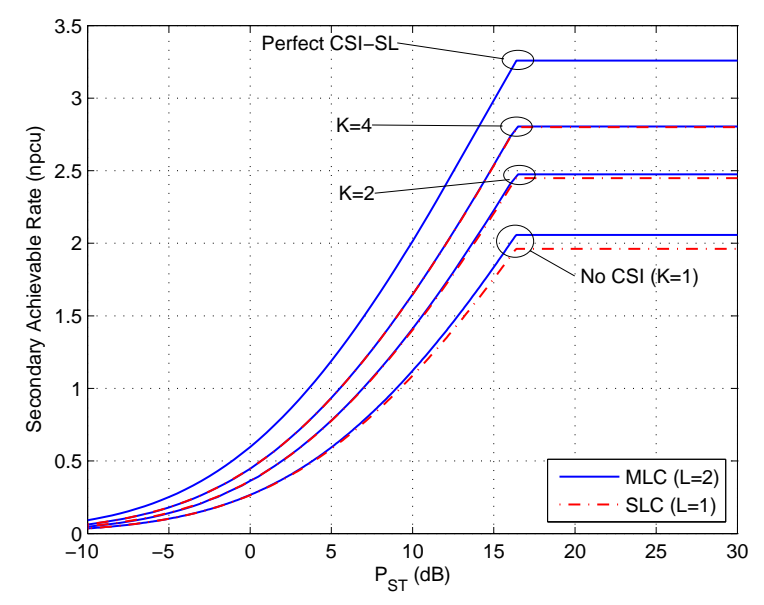

(a) Non-cooperative Rate (Imperfect CSI-CL).

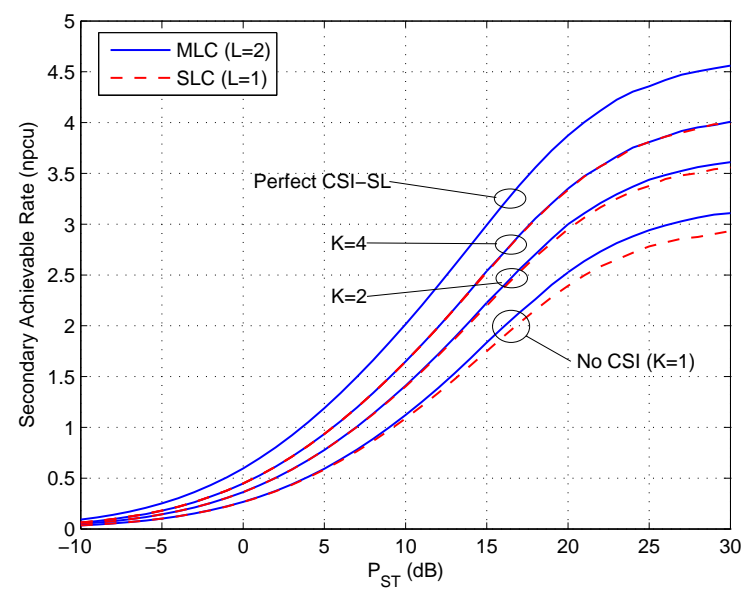

(b) Cooperative Rate (Perfect CSI-CL).

Figure 6: Average achievable rate by the secondary user using quantized CSI and multi-layer coding.

We have shown that the secondary rate, with a single-layer coding, is enhanced considerably even with few bits of CSI feedback. Adopting a MLC in this context further improves the throughput and the MLC outperforms again the OA for a given number of feedback. However, as the number of feedback bits increases, the relative improvement of the MLC becomes limited which indicates that there exists a trade-off between the number of layers used in the MLC and the number of feedback bits available at the secondary transmitter in a CR context. Moreover, we have noted that achieving the prefect CSI secondary rate require a high number of feedback bits even with MLC. Finally, we have shown that cooperation enhances the secondary rate only when the power is higher than a certain value that depends on the tolerated interference.

\section{REFERENCES}

[1] L. Sboui, Z. Rezki, and M.-S. Alouini, "Improving the throughput of cognitive radio networks using the broadcast approach," in Proc. of the IEEE Global Telecommunications Conference (GLOBECOM'13), Atlanta, GA, USA, 2013.

[2] "Spectrum policy task force," Tech. Rep. ET Docket no. 02-135, Federal Communications Commission, Nov. 2002. 
[3] X. Hong, J. Wang, C.-X. Wang, and J. Shi, "Cognitive radio in 5G: a perspective on energy-spectral efficiency trade-off," IEEE Communications Magazine, vol. 52, pp. 46-53, July 2014.

[4] A. Goldsmith, S. Jafar, I. Maric, and S. Srinivasa, "Breaking spectrum gridlock with cognitive radios: An information theoretic perspective," Proceedings of the IEEE, vol. 97, pp. 894-914, May 2009.

[5] J. Mitola and G. Q. Maguire, "Cognitive radio: Making software radios more personal," IEEE. Personal Communications, vol. 6, pp. 13-18, Aug. 1999.

[6] D. Tse and P. Viswanath, Fundamentals Of Wireless Communication. Cambridge Press, 2005.

[7] M.-S. Alouini and A. Goldsmith, "Capacity of Rayleigh fading channels under different adaptive transmission and diversity-combining techniques," IEEE Trans. on Vehicular Technology, vol. 48, pp. 1165-1181, Jul. 1999.

[8] G. Caire and S. Shamai, "On the capacity of some channels with channel state information," IEEE Trans. on Information Theory, vol. 45, pp. 2007-2019, Sep. 1999.

[9] L. Ozarow, S. Shamai, and A. Wyner, "Information theoretic considerations for cellular mobile radio," IEEE Trans. on Vehicular Technology, vol. 43, pp. 359-378, May 1994.

[10] G. Caire, G. Taricco, and E. Biglieri, "Optimum power control over fading channels," IEEE Trans. on Information Theory, vol. 45, pp. 1468 1489, July 1999.

[11] S. Verdu, "Spectral efficiency in the wideband regime," IEEE Trans. on Information Theory, vol. 48, pp. 1319-1343, June 2002.

[12] S. Jin, M. McKay, K.-K. Wong, and X. Li, "Low-SNR capacity of multiple-antenna systems with statistical channel-state information," IEEE Trans. on Vehicular Technology, vol. 59, pp. 2874-2884, July 2010.

[13] X. Li, S. Jin, M. McKay, X. Gao, and K. kit Wong, "Capacity of MIMOMAC with transmit channel knowledge in the low SNR regime," IEEE Trans. on Wireless Communications, vol. 9, pp. 926-931, March 2010.

[14] C. Zhong, S. Jin, K.-K. Wong, M.-S. Alouini, and T. Ratnarajah, "Low SNR capacity for MIMO Rician and Rayleigh-Product fading channels with single co-channel interferer and noise," IEEE Trans. on Communications, vol. 58, pp. 2549-2560, Sep. 2010.

[15] Z. Rezki and M.-S. Alouini, "On the capacity of Nakagami-m fading channels with full channel state information at low SNR," IEEE Wireless Communications Letters, vol. 1, pp. 253-256, June 2012.

[16] D. Love, R. Heath, V. Lau, D. Gesbert, B. Rao, and M. Andrews, "An overview of limited feedback in wireless communication systems," IEEE Journal on Selected Areas in Communications, vol. 26, pp. 1341-1365, Oct. 2008.

[17] T. Cover, "Broadcast channels," IEEE Trans. on Information Theory, vol. 18, pp. 2-14, Jan. 1972.

[18] S. Shamai and A. Steiner, "A broadcast approach for a single-user slowly fading MIMO channel," IEEE Trans. on Information Theory, vol. 49, pp. 2617-2635, Oct. 2003.

[19] T. Kim and M. Skoglund, "On the expected rate of slowly fading channels with quantized side information," IEEE Trans. on Communications, vol. 55, pp. 820-829, Apr. 2007.

[20] D. Tse, "Optimal power allocation over parallel Gaussian broadcast channels," in Proc. of the IEEE International Symposium on Information Theory (ISIT'97), Ulm, Germany, p. 27, Jun-Jul 1997.

[21] K. Huang and R. Zhang, "Cooperative feedback for multiantenna cognitive radio networks," IEEE Trans. on Signal Processing, vol. 59, no. 2, pp. 747-758, 2011.

[22] X. Chen, Z. Zhang, and C. Yuen, "Adaptive mode selection in multiuser MISO cognitive networks with limited cooperation and feedback," IEEE Trans. on Vehicular Technology, vol. 63, pp. 1622-1632, May 2014.

[23] L. Sboui, H. Ghazzai, Z. Rezki, and M.-S. Alouini, "Achievable rate of a cognitive MIMO multiple access channel with multi-secondary users," IEEE Communications Letters, vol. 19, pp. 403-406, Mar. 2015.

[24] P. Schramm, "Multilevel coding with independent decoding on levels for efficient communication on static and interleaved fading channels," in Proc. of the 8th IEEE International Symposium on Personal, Indoor and Mobile Radio Communications (PIMRC '97), Helsinki, Finland, vol. 3, pp. 1196-1200, Sep 1997.

[25] M. Hossain, P. Vitthaladevuni, M.-S. Alouini, V. Bhargava, and A. Goldsmith, "Adaptive hierarchical modulation for simultaneous voice and multiclass data transmission over fading channels," IEEE Trans. on Vehicular Technology, vol. 55, pp. 1181-1194, Jul. 2006.

[26] L. Sboui, Z. Rezki, and M.-S. Alouini, "Capacity of cognitive radio under imperfect secondary and cross link channel state information," in Proc. of the 22nd IEEE International Symposium on Personal Indoor and
Mobile Radio Communications (PIMRC'11), Toronto, Ontario, Canada, pp. 614-618, Sep. 2011.

[27] A. Sendonaris, E. Erkip, and B. Aazhang, "User cooperation diversity. Part I. System description," IEEE Trans. on Communications, vol. 51, pp. 1927-1938, Nov. 2003.

[28] X. Kang, Y.-C. Liang, A. Nallanathan, H. Garg, and R. Zhang, "Optimal power allocation for fading channels in cognitive radio networks: Ergodic capacity and outage capacity," IEEE Trans. on Wireless Communications, vol. 8, pp. 940-950, Feb. 2009.

[29] L. Musavian and S. Aissa, "Fundamental capacity limits of cognitive radio in fading environments with imperfect channel information," IEEE Trans. on Communications, vol. 57, pp. 3472-3480, Nov. 2009.

[30] Z. Rezki and M.-S. Alouini, "Ergodic capacity of cognitive radio under imperfect channel-state information," IEEE Trans. on Vehicular Technology, vol. 61, pp. 2108-2119, June 2012.

[31] Y. Liu, K. Lau, O. Takeshita, and M. Fitz, "Optimal rate allocation for superposition coding in quasi-static fading channels," in Proc. of the IEEE International Symposium on Information Theory (ISIT'02), Lauzanne, Switzerland, p. 111, June-Jul. 2002.

[32] D. N. Tse, "Optimal power allocation over parallel Gaussian broadcast channels," unpublished, available at: http://www.eecs.berkeley.edu/ dtse/broadcast2.pdf, 2000.

[33] W. Su, J. Matyjas, and S. Batalama, "Active cooperation between primary users and cognitive radio users in heterogeneous ad-hoc networks," IEEE Trans. on Signal Processing, vol. 60, pp. 1796-1805, April 2012.

[34] L. Sboui, Z. Rezki, and M.-S. Alouini, "A unified framework for the ergodic capacity of spectrum sharing cognitive radio systems," IEEE Trans. on Wireless Communications, vol. 12, pp. 877-887, Feb. 2013.

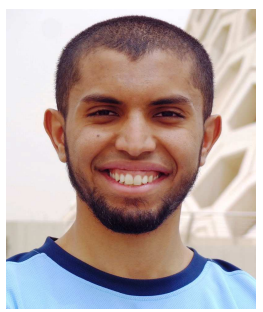

Lokman Sboui (S'11) was born in Cairo, Egypt. He received the Diplome d'Ingénieur degree with honors from Ecole Polytechnique de Tunisie (EPT), La Marsa, Tunisia, in 2011, the MS degree from King Abdullah University of Science and Technology (KAUST) in May 2013. He is currently a Ph.D. candidate in the Electrical Engineering program of KAUST. His current research interests include: performance of cognitive radio systems, low SNR communication, MIMO communication, Relaying performances, energy efficient power allocation and green wireless sensor networks.

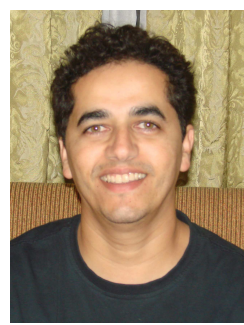

Zouheir Rezki (S'01, M'08) was born in Casablanca, Morocco. He received the Diplome d'Ingénieur degree from the École Nationale de l'Industrie Minérale (ENIM), Rabat, Morocco, in 1994, the M.Eng. degree from École de Technologie Supérieure, Montreal, Québec, Canada, in 2003, and the Ph.D. degree from École Polytechnique, Montreal, Québec, in 2008, all in electrical engineering. From October 2008 to September 2009, he was a postdoctoral research fellow with Data Communications Group, Department of Electrical and Computer Engineering, University of British Columbia. He is now a research scientist at King Abdullah University of Science and Technology (KAUST), Thuwal, Makkah Province, Saudi Arabia. His research interests include: performance limits of communication systems, cognitive and sensor networks, physicallayer security, and low-complexity detection algorithms.

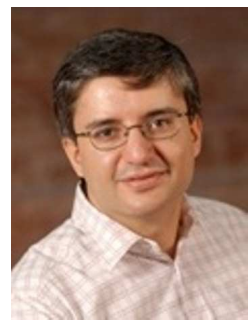

Mohamed-Slim Alouini (S'94, M'98, SM'03, F'09) was born in Tunis, Tunisia. He received the Ph.D. degree in Electrical Engineering from the California Institute of Technology (Caltech), Pasadena, CA, USA, in 1998. He served as a faculty member in the University of Minnesota, Minneapolis, MN, USA, then in the Texas A\&M University at Qatar, Education City, Doha, Qatar before joining King Abdullah University of Science and Technology (KAUST), Thuwal, Makkah Province, Saudi Arabia as a Professor of Electrical Engineering in 2009.

His current research interests include the modeling, design, and performance analysis of wireless communication systems. 